\title{
Discussion of How to Write Business Letters in English on a Basic Level
}

\author{
Yifan Zhang \\ College of Humanities \& Sciences of Northeast Normal University \\ Changchun City Jilin Province 130024
}

Keywords: Business writing in English; Letter; Writing skills

\begin{abstract}
For those people who have just graduated from high school and become a major of Business English in a college, and who have just started a job involving dealing with correspondence in English, they have to know the fact that it`s not easy to use English language to write a letter in a concise manner with appropriate expression and correct format. There`s a huge difference between writing a business letter in English and writing a composition in English. The impression given to the reader by style of language and structure of text has a direct influence on the business of the company. The most of the companies which require their staff to write business letters in English usually have foreign trade business, or are government organizations, therefore, every letter they write is important! A well-written business letter in English may lead to a huge transaction, or an agreement of cooperation in certain business areas between two countries; a badly-written business letter, however, may lead to a big financial loss. The discussion in this paper will include the basic principles of writing a business letter in English, with the hope to help the younger learners to improve their writing skills.
\end{abstract}

\section{Introduction}

In China, writing letters for daily business is a challenge for both English learners and the people using English as a tool for communication in their workplace, so the requirements of using appropriate words and expressions, and ensuring of proper text structure will level up the difficulty of writing a business letter in English.

Correspondence is one of the frequently-used ways for communication in international business trade, therefore, the proper understanding of appropriate words and correct format of the business letter is very important for the communication in the field of international trade. English is not the official language of China. Although the government has put a great deal of human resources and financial resources into the English education, the average level of English language ability in the country is still inadequate. The development of the college major of Business English hasn't run its full course, and there`s a scarcity of the talents who are able to communicate effectively with foreigners by writing business letters in English, and many companies need this type of talent desperately.

International trade business is usually accompanied by intercultural communication, in which process the mutual trust between two parties will be accumulated gradually. The letters for business communication can be saved for a long time, which makes them easily accessible. The more important the communication is, the more likely they will be in the form of correspondence. In the communication in the form of English business correspondence, not only will the correct grammar be required, but also the formal expression, precise wording, concise structure and correct format will be required, by which, formality and business etiquette will be presented. Therefore, every letter sent to customers will give them an impression of the company. even may make or lose a business deal.

In the following part, I will discuss the key factors determining a proper business letter in English. 


\section{Object of Writing}

The first thing you have to do in the communication is to learn about the person you re talking with, especially in the intercultural communication. It would help to break the ice in the beginning of the communication if we learn more about the country, culture, gender, personality, occupation, family situation of the person we re sending letters to, making related affairs dealt with more smoothly, otherwise, a taboo may be violated unintentionally, resulting in a failure of gaining a business.

I’ve met a black customer from Denmark before, after knowing him for a while, I found that he never mentioned his family in front of me, I wanted to ask him about it, but I didn't, because I figured it might be better to learn of his situation from other colleagues first. And it turned out that he was a gay, and easily to be offended when other people asked him about his marriage, if he had a wife or any children, etc. It was a good thing that I didn't ask him about his marriage. Later on, I'd learned that his hometown was Zambia in Africa, and I had been there. So, in a business letter written in English that I sent to him, I added a greeting in Bamba language spoken in Zambia at the beginning, which gave him a big surprise, since then, we had found many common interests, and made many business deals for our companies.

\section{Wording}

There re three requirements to ensure of appropriate wording in a business letter written in English.

First, do not use ambiguous words, the selected words should serve for stating facts. This requirement is aiming to ensure of the efficiency of communication, in other words, to make the letter understandable to the reader without misunderstanding, which purpose may be defeated by using ambiguous words or phrases. For example, 07/12/1975 can be written as 1975, Jul. 12th, as an American expression, and can also be written as 1975, 12th Jul., as a British expression, therefore, the most proper expression would be Jul. 12, 1975.

Second, use formal vocabulary to avoid informal expression used in daily conversation. For example: "quotation" should be used instead of "price list"; "take out a policy" should be used instead of "buy an insurance". This requirement is aiming to ensure of the formal expression, the writing style in a business text should take on the formality with precise expression, to level up the image of the company.

Third, think like a native speaker when choosing appropriate words and expressions. For example: I had a friend working in a foreign-affair-related company, and his boss doesn`t understand English, one day, his boss asked him to translate a letter into English, and my friend did it, at the end of the letter, my friend added " Yours Faithfully" as a customary expression in an English letter, but his boss considered this expression to be inappropriate and asked him to exchange these words to another expression.

Short sentences are preferred in a business letter, the length of a sentence is usually within fifteen or eighteen words, otherwise, the text may be difficult for the reader to understand, in which case, the reader may overlook some information leading to a misunderstanding. Therefore, in the translation from Chinese to English, a long translated sentence should be divided into several short sentences. Of course, long sentences and short sentences should exist together to keep the structure from being too dull. In the business writing, inverted sentences and parenthetical sentences can be used to emphasize the important points.

\section{Arrangement of Paragraphs}

Nowadays, people prefer to read in the manner of grasping the most important information by skimming a huge amount of text materials. Although some disadvantages are accompanied with this way of reading, but it`s still an efficient approach to gain information, which should be taken into account when writing a business letter, because people you work with may not have enough time to read a long piece of text. A decent business letter should have a proper structure with emphasis on the important points. 
The structure can be achieved by that: (1) the first and last paragraphs must feature in the important points, with a proper length of 1 to 3 lines; (2) the paragraphs in the middle part can be divided into different levels by adding secondary titles, emphasizing the important points. The length of each paragraph should be kept within 8 lines as much as possible, otherwise it may influence the understandability of the text.

\section{Pleasant Appearance}

Last but not least, the pleasant appearance of a text cannot be neglected. The human cognitive system is a multi-dimensional complex, besides rational judgement, emotional judgement will also be made by human for the most of times. The text with a pleasant feeling at the first sight will help to put across the information, making it easier to be understood by the reader, which requires:

(1) Correct format. The format of English letter is different from that of Chinese letter, which will not be elaborated here. In short, the format in the cover and the main part should be taken into account when writing a letter in English, especially when it`s a business letter, the format must be correct.

(2) Sloppy handwriting should be avoided when it`s a written letter, and typeface should be visually pleasant when it's a printed letter. Bold font and italic font can be used to emphasize the words with important information, but they cannot be used too often, otherwise the text will come out too messy, and the words treated by the font will not be obvious. Finally, special attention should be attached to the selection of proper type size, line spacing and page margin.

These are some opinions of my own about writing business letters in English, it`s inevitable that there might be some aspects of this subject omitted. I hope this paper will give the learners in need for help some beneficial ideas.

\section{References}

[1] Techniques of Writing Business Letters in English_Baidu Wenku http://wenku.baidu.com/view/63acb20d6c85ec3a87c2c598.html?re=view

[2] Xiaoli Hou. Exploration of Teaching Method of Writing Business Letters in English[J]. Northern Economy and Trade, 2004,(2)

[3] Fang Tan, Huiping Su. Foreign Business English Correspondence[M]. Beijing: Xuelin Press, 2005 